\title{
La représentation inversée des valeurs et normes féminines dans Jacky au royaume des filles de Riad Sattouf
}

\author{
Nathalie Rouphael \\ Université Saint-Joseph, Liban
}

\section{Introduction}

Il ne devrait plus être nécessaire aujourd'hui de justifier un film comme objet scientifique (Diken et Laustsen 7), digne de considération et d'analyse au même titre que le texte littéraire ou l'œuvre picturale. De surcroît, le film ne se limite pas à son statut d'objet d'art isolé de son contexte de production; les films sont «des objets immanents, éventuellement redevables d'une lecture transcendantale », et le film n'est compréhensible « qu'en raison du statut social qu'il acquiert » (Esquenazi 15). Nous démontrerons donc comment Jacky au royaume des filles (dorénavant $J R F$ ) est le produit d'un milieu social et politique aussi légitime que toute autre production intellectuelle ou artistique. Pour ce faire, nous débuterons par une présentation succincte de l'auteur, de la réception $\mathrm{du}$ film auprès du grand public et des acteurs car leur choix a une importance directe sur les représentations et le discours du film. Nous enchaînerons par un positionnement théorique qui nous placera dans le courant des Queer Studies, et qui élimine donc toute approche esthétisante de l'analyse. Notre vision n'est pas unidisciplianire, mais le cadre et les limites de cet article ne permettent pas une analyse multidisciplinaire exhaustive. C'est donc une grille sémiologique qui sera appliquée à la lecture du film, en tant qu'objet culturel, social et partiellement historique.

Par la suite, nous tenterons d'évaluer les représentations des hommes dans le film, et donc indirectement celles des femmes telles que véhiculées par l'auteur. Nous aborderons la dichotomie entre féminin et masculin dans le pays imaginaire de Bubunne, aux niveaux politique, militaire, vestimentaire, alimentaire, linguistique et sexuel, pour terminer par une brève analyse d'une séquence représentative du film. La présentation de ces représentations nous permettra d'établir des liens entre Le Deuxième Sexe et $J R F$, afin de montrer que le premier n'a malheureusement pas encore totalement perdu de son actualité. Enfin, nous conclurons en défendant un point de vue intersectionnel, notion qui sera alors définie et défendue dans le champ de la cause féministe. Le film, sa réception et ses acteurs Riad Sattouf est un auteur de bandes dessinées et un réalisateur franco-syrien, né à Paris en 1978. Ayant passé une partie de son enfance en Libye et Syrie, il témoigne : « Les femmes c'était ou une mère ou une tante habillée en noir, une grand-mère habillée en noir, ou des petites filles avec qui on n'avait jamais de contact, qui passaient comme ça. Sauf des contacts d'insulte » (Sattouf Clique).

Marqué par cette expérience, les rapports entre les hommes et les femmes, la sexualité naissante ou la déconstruction des stéréotypes genrés sont des thèmes récurrents dans les œuvres de Sattouf. Son premier film en tant que réalisateur, Les Beaux Gosses (2009), traitait de la puberté, des mutations du corps, du désir sexuel et de la recherche de soi, sur un ton burlesque mais néanmoins poétique, offrant un panorama paradoxalement drôle du spleen adolescent. Les Beaux Gosses fait 900.000 entrées en France, ce qui est une réussite honorable pour un premier film. Il est également très bien accueilli par la presse et les critiques qui saluent son discours genré très critique ; le public 
découvre Vincent Lacoste, qui sera choisi pour interpréter le héros de $J R F$. Malgré ce premier succès, $J R F$ sera un échec commercial et critique. La faute n'est pas à imputer au casting : Vincent Lacoste et Charlotte Gainsbourg portent admirablement le film, et quelques seconds rôles sont interprétés par des personnalités appréciées et connues pour leur côté satirique (Didier Bourdon, Anémone, Michal Hazanavicius). Le public a peut-être constaté une trop grande noirceur dans un film présenté comme une comédie jugée trop dérangeante, tandis que Riad Sattouf explique l'échec du film par le clivage que connaissait la société française à l'époque : «Il est sorti à l'époque des manifs contre le mariage pour tous, dans un contexte de crispation générale. Les féministes ont détesté, les antiféministes m'ont envoyé des lettres d'insultes... C'était terrible » (Braunstein n.p.). Tel que nous le verrons, des scènes particulièrement difficiles surgissent en effet en plein milieu d'instants plus légers. Comme dans la vie réelle où le tragique peut survenir à tout moment, ces scènes en arrivent à mieux dénoncer ce à quoi s'attaque le film, fût-ce au prix d'un succès populaire.

\section{Cadre théorique}

Il existe différents points de vue sur la raison d'être du cinéma : il peut être divertissement, outil de communication ou expression artistique (Lever). D'autres lui ont recherché des missions plus sociales ou politiques. Ainsi Erwin Panofsky, André Bazin et Stanley Cavell ont démontré, chacun à sa façon, que le cinéma entretient un lien singulier à la réalité. Il ne s'agit pas de représenter au cinéma la réalité en la copiant ou en la reproduisant selon un vain principe de fidélité. Inversement, même les films traitant de mondes fantastiques a priori très éloignés du monde « réel », ne sont pas « déconnectés » du réel car le lien que nous évoquions plus haut n'est justement pas un lien de représentation dont la valeur dépend de la qualité de cette dernière. Paola Marrati souligne justement que le cinéma n'est donc pas strictement représentatif (du réel), car toute représentation est subjective (Marrati 66). Dès lors, il serait insignifiant d'apposer une quelconque limite à la subjectivité d'un auteur, et la nature du lien entre ce qui est représenté à l'écran et la réalité reste la même.

Précisons néanmoins que les films ne constituent pas pour autant des documents historiques. "La théorie du reflet» de Yann Darré, dite réflexionnisme (Bordwell, Thompson et Ashton) stipule que tout film est un miroir de son époque, y compris et même surtout si son auteur s'en défend; mais elle rencontre un obstacle de représentativité, formalisé par Yann Darré comme suit :

Épistémologiquement, la théorie du reflet [le cinéma comme reflet d'une société] rencontre deux obstacles. L'un est affronté par les auteurs et se traduit dans un choix : faut-il étudier tous les films d'une période ou seulement les œuvres les plus importantes, le talent et la postérité garantissant le caractère représentatif de l'œuvre. L'autre obstacle est généralement ignoré, ou contourné grâce à l'argument de la reconnaissance (à tous les sens du terme) par le public: de quelle fraction de la société les cinéastes sont-ils représentatifs ? (Darré Esquisse 128)

La théorie du reflet ne résiste pas non plus au prisme de la mise en scène qui altère toute possibilité de "miroir»: la réalité ne peut être montrée mais représentée, tel que le formulent Bülent Diken et Carsten Bagge Lausteen : 
In a sense, cinema functions as a kind of social unconscious : it interprets, invents, displaces, and distorts the object of sociological inquiry. What films offer is not just a reflection on society; they are part and parcel of the society they portray. Cinema does not, however, only mirror/distort an external reality but also opens up the social world to a vast domain of possibilities. Cinema is often an experiment with changing social forms (Diken et Laustsen 4-5)

Si nous concluons que toute volonté de « film miroir » n'est plus aujourd'hui concevable, il sera intéressant d'examiner ce que représente un film dont l'auteur lui-même revendique le statut de « miroir inversé » (Brinaire n.p.). Pour l'heure, nous considérerons le film comme élément inhérent à la culture d'une société (Ferro; Sorlin), en tant que produit d'un champ social et culturel luimême tributaire des conditions historiques de son époque.

Art et féminisme. Le Deuxième Sexe paraît en 1949, mais il fallut attendre une vingtaine d'années avant que les liens entre art et féminisme ne se tissassent véritablement. C'est en effet à l'orée des années 1970 que le féminisme, en tant que mouvement politique, s'entrecroise avec le champ culturel et le nourrit, non seulement dans son expression, mais aussi chez les théoriciens et historiens de l'art qui intégrèrent les outils proposés par les théories féministes dans les domaines de l'art et des sciences humaines.

Dans le féminisme, et donc politiquement, les œuvres considérées comme politiques sont celles qui considèrent la féminité comme une catégorie sociale à part entière, faisant fi, dans le cadre de l'œuvre, des rapports de force et de domination. C'est exactement cette tendance que l'on retrouve dans la périodes des années 1970, tendance qui milite pour l'autonomie des femmes et l'instauration de rapports égalitaire entre les hommes et les femmes en s'appropriant des artefacts, des signes, des formes et des techniques historiquement connotés, pour mettre à nu et détruire les stéréotypes d'identité sexuelle (Beaudry). Parmi ces œuvres, Lucille Beaudry évoque par exemple $L a$ chambre nuptiale de Francine Larivée au Québec, et il est frappant de constater que son analyse pourrait en tout point s'appliquer à $J R F$ :

L'œuvre s'en prend aux relations traditionnelles entre les hommes et les femmes dans le couple et au sein de la famille, sans épargner la vacuité de la société de consommation et l'atavisme des valeurs religieuses qui ont longtemps façonné la société québécoise, en particulier la place des femmes au sein de la famille traditionnelle et dans la société (Beaudry 11)

Il conviendra, pour une histoire plus détaillée du féminisme et de ses courants, de consulter Thébaud, Riot-Sarcey ou Fraser \& Ferrarese, mais nous citerons toutefois Elvan Zabunyan, pour qui deux ouvrages marquent le champ entre féminisme et art: The Dialectic of Sex de Shulamith Firestone (1970) et Sisterhood is Powerful, anthologie dirigée par Robin Morgan (1970) et premier recueil d'écrits féministes publié aux États-Unis, qui deviennent des références dès leur parution. Elvan Zabunyan écrit par ailleurs :

Ce tournant des années 1970 apparaît dès lors comme un moment central où l'analyse des pratiques artistiques va pouvoir se faire selon une méthodologie ramenant la figure de la femme à une place stratégique et permettant à des notions propres au champ des arts visuels et, en premier lieu, celle de la représentation, d'être interrogées différemment 


\section{(Zabunyan 172)}

Concrètement, ces évolutions donnaient lieu à la naissance des Queer Studies, qui sont la troisième vague des Cultural Studies. ${ }^{1}$ La démarche des Queer Studies consiste à étudier le poids des normes hétéronormatives sur les personnes dont le corps et les désirs ne s'accordent pas aux standards dominants. Souvent interventionnistes, les Queer Studies s'intéressent à la construction des identités sexuelles pour les remettre en cause, systématiquement sur le principe de rupture entre l'identité et une quelconque essence, et donc en résonnance avec la construction de l'identité féminine telle que définie dans Le Deuxième Sexe.

Nous proposons ainsi d'examiner le film $J R F$ à la lumière de ces quelques éléments théoriques ; en résumé, nous le voyons comme un produit d'un contexte géopolitique difficile voire douloureux, d'une situation sociale de la femme et du regain des extrémismes religieux de par le monde. Il cristallise également les propres manifestations de l'inconscient collectif de son auteur, mais nous nous limiterons à une approche globale et sociale. Les limites de cet article nous interdisent une approche véritablement pluridisciplinaire, nous adopterons donc celle des Queer Studies, qui consistera à analyser la représentation (en l'occurrence inversée dans $J R F$ ) de la femme, des valeurs féminines d'une part, et à commenter ce que l'inversion totale des normes voulue par Riad Sattouf peut apporter à la cause féministe. Si les Queers Studies sont parfois critiquées pour leur incapacité à analyser un film sur le plan purement cinématographique, nous revendiquons et assumons pleinement cette approche : nous ne traiterons pas d'esthétique, nous limitant à une analyse du fond délaissant la forme cinématographique. Cette dernière n'est que très faiblement au service du contenu, notamment en raison des choix du réalisateur et auteur Riad Sattouf qui a opté pour une réalisation épurée, simple et efficace, très peu esthétisante. Elle est en revanche chargée d'un point de vue sémiologique, tel que nous le verrons.

\section{L'inversion des normes féminines et sexuelles dans JRF}

$J R F$ paraît en 2014, dans un contexte géopolitique international complexe ; la peur du terrorisme islamiste règne en Occident et le printemps arabe a débuté trois ans plus tôt. La proclamation de l'État islamique, cette même année, sur les territoires de la Syrie et de l'Irak, pourrait triplement illustrer les fléaux que veut dénoncer Sattouf dans son film, à savoir le politique, le religieux et le social ; en effet, l'État islamique est une dictature religieuse où les femmes sont réduites au statut d'esclaves sexuelles (Cojean), pouvant être vendues sur les marchés. Il convient de préciser que Riad Sattouf a toutefois commencé à écrire son scénario en 2009, avant les événements que nous évoquons ici. Malgré tout, le film ne pourrait être coupé du contexte historique de son année de parution, ne serait-ce qu'en raison de la réception auprès de téléspectateurs eux-mêmes pleinement acteurs de ce contexte. Nous sommes ici en accord avec Sorlin, qui écrit qu'il s'agit d'étudier « non ce que le film veut exprimer mais ce qu'il dit » (Sorlin 268)

Le film est écrit et réalisé par Riad Sattouf. Sur un modèle orwellien revendiqué, il imagine une dystopie gynocratique, dictatoriale et fortement

${ }^{1}$ Pour le détail historique et théorique des Cultural Studies, voir Neveu et Mattelart. Introduction aux cultural studies. Paris : La Découverte, 2010. 
religieuse, où les hommes souffrent de la domination des femmes. Il s'agit de Bubunne, pays imaginaire gouverné d'une main de fer et où la seule religion repose sur la communication télépathique avec des chevaux. Se voulant également une réadaptation de Cendrillon, $J R F$ conte tout particulièrement le parcours de Jacky, jeune homme pauvre en âge de se marier, et amoureux de la Colonelle Bubunne XVII, fille de Bubunne XVI (au pouvoir depuis 34 ans), qu'il espère épouser lors du traditionnel «Bal de la Grande Bubunnerie », occasion à laquelle la Colonnelle choisira son « grand Couillon ». Après la mort de sa mère, Jacky vivra chez sa tante où il sera persécuté et empêché d'aller au Bal. C'est avec l'aide de Julin, un ami prostitué de son défunt père, qu'il pourra, à l'instar de la fable de Cendrillon, rejoindre le Bal et conquérir le cœur de la Colonelle Bubunne. JRF n'est pas un film qui imaginerait ce que serait le monde si les femmes étaient au pouvoir, mais une sorte d'antireflet de la société réelle.

L'inversion des rôles, comédie ou drame? Si le résumé du film prête à sourire, $J R F$ est effectivement jalonné de situations comiques, qui découlent essentiellement de l'inversion des rôles. En instaurant un matriarcat pur et dur, les hommes sont voilés, couards, faibles, mesquins, jaloux, fascinés et sexuellement attirés par le pouvoir et l'autorité. Ces poncifs, habituellement attribués à la femme, sont inversés avec un humour volontaire, et l'on pourrait supposer que JFR se limite à être une comédie parodique. Pourtant, l'humour, s'il est plus ou moins présent selon les sensibilités de chacun, est presque systématiquement mis à mal par des séquences poignantes, dérangeantes, voire cyniques. JRF comporte ainsi plusieurs exécutions (par pendaison et par fusillade), l'exploitation sexuelle d'une dominante (ici la femme) sur un dominé en échange de marchandises, un début de viol collectif ( 3 femmes militaires violant Jacky attaché à un arbre), la mort de sa mère sous les yeux de Jacky (où une comédie classique aurait laissé Jacky faire ses adieux à sa mère). Il convient alors de se demander si, dans $J R F$, les rires sont entrecoupés de ces moments sombres et gênants, ou l'inverse. Pour l'auteur, le désir d'équilibre réside sans doute dans l'importance qu'il accorde à la question des inégalités entre les sexes : « [C'est] l'un des sujets les plus importants du monde actuel. Beaucoup plus que la religion, la vraie question aujourd'hui porte sur le droit des femmes à disposer d'une sexualité libre. Tout est lié au genre. " (Blottière n.p.). Concernant ces viols et ces violences que subissent les hommes de $J R F$, Kimberlé Crenshaw écrit :

Ainsi admet-on aujourd'hui que les sévices et le viol, autrefois considérés comme des affaires privées (à régler en famille) et des comportements aberrants (déviance sexuelle), participent d'un vaste système de domination qui touche les femmes en tant que classe. Cette reconnaissance progressive du caractère social et systémique de phénomènes longtemps perçus comme ponctuels et individuels caractérise également la politique de l'identité défendue, entre autres, par les gens de couleur ou les gays et les lesbiennes. Tous ces groupes ont puisé leur force, leur sens de la communauté et leur développement intellectuel dans une politique fondée sur la notion d'identité (Crenshaw 52)

L'intérêt de $J R F$ est de fait politique. Plus ou moins ancré dans l'écrasante majorité des sociétés humaines contemporaines, le rapport des forces favorable à l'homme devient flagrant lorsqu'il est inversé, car il bouscule les habitudes du 
spectateur et de la spectatrice, et le procédé participe alors à une prise de conscience plus élaborée. Le dialogue opéré entre le burlesque ou le fantaisiste d'une part, et le tragique d'une société opprimée à tous les niveaux d'autre part, rend effroyables les situations comiques et humoristiques les aberrantes inégalités entre classes et entre sexes ; en ceci, $J R F$ est constamment alimenté d'une charge émotive qui permet à son auteur de nous conter son histoire et de nous dresser une liste des turpitudes sociales qu'il désire dénoncer.

Le pays imaginaire de JRF. Le pays imaginaire est nommé Bubunne, Riad Sattouf explique que l'objectif était de « prendre un mot ridicule et de le sacraliser. Les personnages devaient considérer avec le plus grand sérieux ce qu'une société comme la nôtre juge complètement débile » (Sattouf 2014). Si l'auteur affirme avoir voulu s'éloigner du modèle moyen-oriental, il admet que son enfance en Syrie l'a certainement marqué. Pays alors proche de l'URSS, l'uniformité visuelle est reproduite dans les blocs de maisons identiques, sans âme. Quand tous les hommes (sous-entendu les femmes) vivent dans des lotissements décrépits et rigoureusement alignés au milieu de la boue, les vies humaines sont alors identiques. Elles ne peuvent plus se construire librement mais sont essentialisées par les valeurs dominantes.

En République de Bubunne, le vice a été poussé jusqu'à l'uniformisation de la nourriture. Tout le peuple consomme une bouillie blanchâtre produite à partir des excréments de la cité. Pour la préparer, il est nécessaire de la « touiller », prérogative évidemment réservée aux hommes, qui se transmettent l'art de touiller de père en fils. Symboliquement, la bouillie représente, à l'instar de l'uniformisation des habitats, l'écrasement de l'individu et donc de toute possibilité d'émancipation, mais il faut surtout y lire une critique de la société de consommation; quand tout le monde produit et consomme une même bouillie tiède et incolore, et qui en l'occurrence est produite à partir des excréments, la pensée unique a réussi son pari. L'un des crimes de Bubunne est d'ailleurs de cultiver des plantains, à savoir toute forme de légume, activité primaire pourtant fondatrice des civilisations humaines. En ce sens, la hiérarchie entre les genres et les dictatures religieuses ou politiques nous privent de notre humanité.

Sur le plan de la religion, les habitants de Bubunne vénèrent les chevaux. L'emblème de Bubunne représente deux têtes équines rouges, dont la forme peut rappeler celle de l'appareil reproducteur féminin. Il s'agit là aussi d'un bousculement de nos habitudes, le cheval étant habituellement symbole de virilité et de fougue masculine. Il est ici associé à la femme, quand ce sont de petits poneys sales qui représentent les hommes.

Les vêtements. Selon leur classe sociale, les femmes de Bubunne portent soit l'uniforme militaire, soit l'uniforme des ouvrières. Quant aux hommes, ils portent tous une sorte de tunique appelée "voilerie » qui ne peut que faire penser à la burqa, au niqab ou au chador. Tout est fait pour avilir l'homme : le bonnet appuie sur la tête et l'homme courbe alors l'échine; la voilerie couvre l'ensemble du corps et le noie dans du tissu, ne laissant paraître que le visage ; l'homme est uniformisé, faible et dépendant. Image de la soumission la plus totale, la voilerie a un anneau vide pour les célibataires, plein pour les hommes mariés. C'est par cet anneau que l'homme est « tenu », et c'est cet anneau que les pères présentent à la Colonnelle dans l'espoir qu'elle s'en emparera, s'appropriant son porteur. En portant cet anneau et cette laisse, l'homme de Bubunne ne possède plus son corps, qui devient propriété de sa femme. Toutes les classes sociales de Bubunne, femmes et hommes, reproduisent la peur d'une 
confusion entre les identités genrées (Maugue); Détrez écrit à ce propos : « Parler de construction sociale rompt ainsi avec l'expérience ontologique à la fois individuelle et commune qui contribue à poser le corps comme expression naturelle de la personne » (Détrez 4).

Sattouf affirme que la voilerie renvoie au voile musulman, mais aussi aux voiles des bonnes sœurs ainsi qu'aux habits des moines bouddhistes en raison de la couleur, et les références sont ainsi noyées. Toutefois, il serait hypocrite de ne pas y voir essentiellement une charge contre le port imposé du voile, ou de tout autre vêtement qui emprisonne, stigmatise ou dépossède le corps. Le vêtement, dont la symbolique première est d'exprimer au regard de l'autre une identité sociale et sexuelle, devient alors militant dans $J R F$, comme il l'est dans la vie réelle.

Le langage. JRF étant un conte orwellien, il possède sa propre novlangue. Les termes renvoyant à des concepts forts ou de domination ont été féminisés, comme blasphèmerie pour blasphème, voilerie pour voile, argenterie pour argent. À l'inverse, des termes péjoratifs ou dégradants ont été masculinisés, comme culottin pour culotte ou sodomage pour sodomie. Sattouf explique certaines exceptions comme forêterie ; l'auteur a voulu souligner le féminin du mot au lieu de l'inverser car la forêt représente un inconscient qui terrorise la société de Bubunne. Nous avons ici une parfaite illustration, qui passe par la dérision et le détournement des rôles, du «sexage», soit de «l'expression de l'idéologie naturaliste sexiste en français» (Michard 17). Dans son ouvrage, Claire Michard conçoit le langage comme l'expression fondamentale des rapports de force sociaux entre les sexes; dès lors, les catégories féminin et masculin ne sont pas aléatoires mais reproduisent les biais cognitifs et sociaux de la réalité, à l'image de la novlangue de Bubunne créée par Riad Sattouf. En effet, Michard considère que le féminisme et la linguistique se retrouvent dans la vérification de deux postulats : le premier est de considérer « l'oppression et l'exploitation généralisée des femmes par les hommes comme une relation sociale fondatrice de la société ", et le second que cette relation influe directement sur la production sémantique et la langue. Nous proposons ici un relevé que nous supposons exhaustif, dans $J R F$, des néologismes qui illustrent la domination d'un genre sur l'autre :

\begin{tabular}{|l|l|l|l|}
\hline \multicolumn{1}{|c|}{ Terme féminisé } & $\begin{array}{c}\text { Terme qu'il est } \\
\text { censé représenter }\end{array}$ & $\begin{array}{c}\text { Terme } \\
\text { masculinisé }\end{array}$ & $\begin{array}{l}\text { Terme qu'il est censé } \\
\text { représenter }\end{array}$ \\
\hline Salopures & Salauds & Culottin & Culotte \\
\hline Blasphémerie & Blasphème & Sodomage & Sodomie \\
\hline Shampouinerie & Shampoing & Merdin & Merde \\
\hline Magasinnerie & Magasin & Toilettins & Toilettes \\
\hline Voilerie & Voile & Crottin & Crotte \\
\hline Argenterie & Argent & Tétage & Fellation ? \\
\hline Hôtellerie & Hôtel & Plantains & Plante \\
\hline Forêterie & Forêt & Homistes & Féministes \\
\hline Poéterie & Poème & Gueusage & Plèbe \\
\hline Gouinerie & Lesbienne & Marion & Marié \\
\hline
\end{tabular}




\begin{tabular}{|l|l|l|l|}
\hline Annoncerie & Annonce officielle & Couillard & Pénis \\
\hline
\end{tabular}

Fig. 1 Néologismes

La sexualité de Bubunne. Dans JRF, les valeurs sexuelles tournant autour du corps, des désirs et de l'identité sexuelle sont inversées. La femme porte un regard lubrique sur le mollet des hommes, et c'est elle qui profite de sa position sociale ou de son argent pour profiter sexuellement de l'homme. Julin, qui se prostitue, dit à l'une de ses clientes qu'elle ne devait pas s'attacher à lui. Trois femmes violent un homme dans une forêt et l'une d'elles, reproduisant un geste presque phallique avec ses seins, lui impose de « téter».

Quant au corps de l'homme, il est soit totalement caché sous la voilerie (dans le monde réel, cela correspond au fantasme de la possession du corps féminin), soit exposé, dénudé, vendu (dans le monde réel, réduction de la femme à son corps dans les œuvres de représentation visuelle, dans les médias et plus particulièrement dans la publicité).

En ce qui concerne le désir de l'homme envers la femme, Jacky se masturbe dans la scène d'ouverture du film devant la photo de la Colonelle, qui représente le pouvoir et l'autorité. Il est intéressant de souligner que dans une autre scène, le geste de la masturbation se confond avec celui du cirage des bottes de sa mère.

En revanche, le personnage de la Colonelle est plus complexe. La scène finale nous révèle que la Colonelle est en réalité un homme que sa mère au pouvoir a fait passer pour une fille afin d'avoir une héritière. De son côté, brodant sur le thème de Cendrillon, Jacky se déguise en soldate pour pouvoir assister au bal. Les scènes de l'antichambre regroupent donc deux hommes déguisés en femmes (voir infra) ils se marieront même quand leurs identités biologiques ne seront plus un secret. La scène finale révèle donc au peuple que la nouvelle dirigeante est non seulement un homme, mais qu'elle aime aussi un autre homme. L'une des femmes de la foule hurlera alors : «Blasphèmerie ! »; si cette note finale est partiellement ouverte, tout laisse à prévoir que la foule va effectivement se révolter, sans que l'on sache si la révolte vient du travestissement en femme de la Colonelle ou de l'homosexualité du couple royal.

La confusion des genres, du féminisme au queer. Dans la première partie du film, les rôles sont clairement fixés et répartis, et nous pourrions être dans ce que d'aucuns pourraient qualifier de vision binaire, certes inversée, mais caricaturale de la société. Sattouf a néanmoins la lucidité et l'intelligence d'éviter cet écueil à partir du moment où Jacky se déguise (travestit ?) en femme pour pouvoir entrer dans le palais, et nous nous proposons ici de revenir sur une séquence particulièrement représentative du propos général du film. Voici ce qui se passe: Jacky est un garçon déguisé en femme, et la Colonelle est également un garçon déguisé en femme. Quand la Colonelle invite Jacky à s'éclipser du bal, son personnage ignore que Jacky est en réalité un garçon, et Jacky ignore que la Colonelle est en réalité un homme. La Colonelle se croit donc attirée par une femme et il en va de même pour Jacky. La première déclarera: "C'est étrange, je suis attiré par vous comme un aimant. » Le « plantain » que la Colonelle offre à Jacky est une carotte. Il peut s'agir d'une allusion au traditionnel cigare entreposé dans un coffret, mais nous ne pouvons ne pas y lire un symbole phallique, car il ne faut pas oublier que du point de vue de la Colonelle, elle est un homme qui tente de séduire une femme. 
Plus tard, quand les deux aimants auront découvert la vérité l'un sur l'autre, la Colonelle dira à Jacky : « Jacky, je ne sais plus quoi penser, je sais plus ce que j'aime, je sais plus qui je suis, j'ai toujours tellement fait semblant. » Ce à quoi Jacky répond : " Mais moi, je vous aime comme vous êtes, même si vous ne savez pas ce que vous êtes. » La portée du film, qui ne traitait la question du genre que d'un point de vue social et collectif, se concentre dès lors sur l'individu et sa quête personnelle de son identité sexuelle. La situation décrite dans $J R F$ nous semble unique : dans une société gynocratique, deux hommes déguisés en femmes éprouvent une attirance l'un vers l'autre. La situation est confuse, mais cette confusion est le cœur du propos de Sattouf qui brouille les pistes pour mieux affirmer que l'attrait sentimental et sexuel peut s'émanciper des questions de genre.

Avec ce passage, ces deux personnages brisent et recomposent les identités corporelles et sexuelles qui, jusque-là, obéissaient à des clivages tranchés et simples entre femmes et hommes. Grâce à Jacky et à la Colonelle, nous avons une véritable subversion des genres à la manière queer décrite par Butler. Lucille Beaudry établit un parallèle entre identité queer et la diversité des arts féministes qui nous semble ici le bienvenu :

Tout comme l'éclatement des frontières entre les disciplines de l'art a marqué l'implantation des pratiques artistiques, éclatement auquel l'art féministe a fortement contribué, la critique déconstructive de la représentation faite par les femmes artistes aura porté la question de l'identité jusqu'à son éclatement en tant que phénomène social d'une identité homogène des femmes. Cette fois, des activités artistiques puisant dans toutes les disciplines vont illustrer la pluralité des identités, comme si le «genre » devait aussi être déconstruit. Une pluralité identitaire émane alors du dévoilement des différences, mais aussi et surtout seront exprimées ou montrées des possibilités d'en changer en mettant l'accent sur la variabilité historique et actuelle des identités, variabilité trop souvent occultée par le genre féminin comme catégorie sociale hiérarchisée (Beaudry 13)

JRF d'après Simone de Beauvoir. Il n'est évidemment pas possible pour nous d'effectuer une relecture beauvoirienne exhaustive de $J R F$, mais il est frappant de remarquer à quel point les six chapitres de la seconde partie du second tome de Le Deuxième Sexe sont parfaitement illustrés dans $J R F$, à savoir : La femme mariée (Chapitre V); La mère (VI), La vie de société (VII), Prostituées et hétaïres (VIII) ; De la maturité à la vieillesse (IX) et Situation et caractère de la femme (X). Toutes les citations qui suivent proviennent de l'ouvrage.

Si Simone de Beauvoir écrivait en 1949 que «la plupart des femmes, aujourd'hui encore, sont mariées, l'ont été, se préparent à l'être ou souffrent de ne l'être pas " (233), il s'agit de la condition masculine dans $J R F$, chaque homme en âge de se marier n'aspire qu'à devenir "esclave ou vassale [vassal] aux groupes familiaux que dominent pères et frères [mères et sœurs] » (234). Le mariage est la seule voie pouvant assurer la stabilité et un statut social respectable aux hommes de $J R F$, et le mariage y est d'ailleurs monétisé, les femmes payant une dot pour s'acquérir d'un homme et le posséder, cette possession étant symbolisée par l'anneau et la laisse.

Quant à la mère beauvoirienne, elle est représentée par le personnage de Brunu, père dont les seules ambitions sont l'entretien de son foyer et le mariage de ses fils. Si le chapitre VI est consacré à la question de l'avortement 
essentiellement, $J R F$ ne mentionne que le statut social et le rôle des pères dans la société de Bubunne, que Beauvoir résume dans ce chapitre comme suit : « ... la femme n'est qu'une femelle domestique qu'on apprécie selon le travail qu'elle fournit et qu'on remplace sans regret si elle disparait » (417). Brunu, mais aussi Franku, sont également des hommes représentatifs du portrait de la femme âgée dressée dans le chapitre IX (De la maturité à la vieillesse) :

Leur morale est abstraite et formelle comme les impératifs de Kant ; elles prononcent des interdits au lieu de chercher à découvrir les chemins du progrès ; elles n'essaient pas de créer positivement des situations neuves : elles s'attaquent à ce qui est déjà afin d'en éliminer le mal ; c'est ce qui explique que toujours elles se coalisent contre quelque chose : contre l'alcool, la prostitution, la pornographie; elles ne comprennent pas qu'un effort purement négatif est voué à l'insuccès (515)

De même, le père bubunnien est une femme traditionnelle telle que décrite dans le chapitre VII consacré à la vie de société : son foyer est «l'expression de son standard de vie, de sa fortune, de son goût » (419), aspect sur lequel Sattouf joue le thème de Cendrillon quand Jacky est persécuté par sa famille d'accueil riche et aisée. Dans JFR, c'est au père que revient le devoir d'ordonner cette vie mondaine, la femme devant assurer les revenus de la famille, mais surtout jouer le rôle d'arbitre et de régulateur des conflits, rôle dont est exclu l'homme.

Le personnage de Julin est particulièrement intéressant et illustre le chapitre VIII : prostitué dans une société où les femmes lubriques imposent la chasteté aux hommes, il doit se vendre pour pouvoir fuir Bubunne et échapper à sa condition d'homme. Contrairement à la prostituée décrite par Beauvoir, c'est aussi un intellectuel qui pense et questionne sa société, il est l'ouverture sur l'extérieur, le monde libre et se rapproche ici de l'hétaïre. Julin est aussi celui qui conduira la révolte pour la liberté de Jacky, celui qui cultive des plantains et le militant qui distribue des tracts. Dans $J R F$ la prostitution est une voie de libération et Julin est un hoministe.

Enfin, la femme décrite dans le chapitre X (Situation et caractère de la femme) est elle aussi identique à l'homme de Bubunne : elle est prudente et mesquine (les hommes de Bubunne sont couards et leurs ambitions basses, Jacky n'hésite pas à dénoncer son protecteur Julin pour récupérer son supposé trésor), elle n'a pas le sens de la vérité, ni de l'exactitude (les hommes de Bubunne sont exclus des négociations et des questions d'argent, hormis Julin), elle manque de moralité (Brunu et ses fils se complaisent à asservir Jacky et à lui voler son carton d'invitation). En bref, l'homme de Bubunne est résumé comme suit : "Elle est bassement utilitaire, elle est menteuse, comédienne, intéressée...» (518).

Tout comme la femme du monde réel connaît une échappatoire à « l'éternel féminin ", l'homme de Bubunne peut échapper à l'éternel masculin de $J R F$, du moins mentalement, tel que le fait Julin, et tel que le fait partiellement Jacky, en aimant un autre homme. Toutefois, comme nous le disions plus haut, la conclusion du film semble indiquer une vision pessimiste de la société qui se révolte, car peu apprêtée encore à accepter la transgression des genres, l'homosexualité et la liberté des corps.

\section{Conclusion}


C'est Kimberlé Crenshaw, féministe majeure de la Critical Race Theory, qui invente la notion et le terme d'intersectionnalité en 1989. À l'image des membres de l'organisation des féministes lesbiennes radicales noires du Combahee River Collective, actif dans les années 1970 aux États-Unis (Crenshaw), l'intersectionnalité désigne la situation de personnes subissant simultanément plusieurs formes de domination ou de discrimination dans une société, le Combahee River Collective considérant justement que l'oppression n'est presque jamais le résultat d'une seule forme de domination.

$J R F$ illustre parfaitement l'intersectionnalité, au sens où ses hommes (ses femmes) subissent à la fois une dictature militaire, une dictature religieuse et l'oppression du genre opposé. Nous pouvons remarquer que le film peut évoquer la Corée du Nord ou l'État islamique, mais aussi certains milieux occidentaux où la femme subit encore la violence, la discrimination ou le viol.

En ce qui concerne sa mission, il faut mentionner que $J R F$ a été un échec commercial en France et à l'étranger, alors que Riad Sattouf avait connu un succès retentissant avec son premier film, et que la production de $J R F$ est tout à fait correcte. Il ne s'agit donc ni d'un manque de notoriété, ni d'un problème budgétaire, mais bien à la difficulté de réception d'un film qui inverse aussi crûment les rôles entre hommes et femmes. Dérangeant, le film instaure une ambiance tragi-comique cynique où voir une femme violer un homme éloigne des recettes prémâchées habituellement servies au spectateur, quitte à ne pas connaître le succès.

Si commercialement c'est un échec, il est à nos yeux une réussite car le combat féministe que Sattouf s'est imposé ne saurait être complaisant. Il aurait été facile de tomber dans le politiquement correct, surtout quand la cause féministe est souvent accusée de l'être. Or Sattouf réussit à déranger le spectateur, car il questionne en lui ses certitudes et ses zones de confort les plus profondes. Tout cela, nous le rappelons, sans procédé esthétisant (mise en scène particulièrement marqué sur le plan esthétique, à l'image du cinéma de Wes Anderson qui insiste par exemple sur des cadrages et des codes de couleurs extrêmement précis) qui eût limité le propos du film, mais en poussant à l'extrême les caricatures de la femme prédatrice et de l'homme infantilisé et asservi. La finale, semi-ouverte, est également une réussite pour les pistes de réflexion qu'elle permet.

À l'heure de l'État islamique, de la dictature des Talibans, de l'infanticide des filles en Inde, de l'oppression en Corée du Nord ou de l'excision des filles en Égypte, l'approche intersectionnelle de Sattouf donne au combat féministe un trait global et universel qui lui manque parfois. Le film n'est pas un témoignage d'un temps révolu, mais bien d'une situation encore contemporaine.

Nous pouvons même lire, vers la fin du film, l'ébauche de propositions plus ou moins directes. L'évolution des mœurs de la société de la République de Bubunne est verticale, de haut en bas, soit du pouvoir vers le peuple. Cette vision des choses apparaissait déjà sous la plume de Simone de Beauvoir, qui considérait par exemple dans Le Deuxième Sexe que maternité et travail n'étaient pas incompatibles à la condition de la coopération et l'aide de l'État, du pouvoir en place. C'est la Colonelle, après un matricide lourd de symbolique freudienne et craintivement applaudi par les aides de camp de la dictatrice, qui met en place une politique d'émancipation et de libération des hommes. Julin revoit d'ailleurs ses ambitions à la baisse et accepte de donner sa chance au nouveau pouvoir. Il ne s'agit pas pour nous de défendre un simple féminisme 
d'état (Stetson et Mazur 1), surtout si celui-ci est réduit à une parité de façade qui en devient " institutionnalisée » (Bereni et Revillard), mais force est de constater qu'une émancipation strictement bottom-up est illusoire, l'évolution découlant tout autant des classes féminines que du pouvoir. La parité, par exemple, est « rendue possible par la conjonction de mobilisations associatives, de pressions internationales et de l'engagement de quelques femmes dédiées à cette cause dans les sphères gouvernementales et parlementaires », et donc "d'une évolution remarquable des rapports entre acteurs étatiques et non étatiques engagés en faveur de cette cause » (Bereni et Revillard 22). JRF offre d'ailleurs un exemple flagrant d'une volonté émancipatrice contrée par le peuple, pourtant logiquement destinataire de cette émancipation, lorsque la femme de la foule refuse la liberté de corps et d'esprit que leur propose la Colonelle. Plus que jamais, la cause féministe intersectionnelle est tributaire d'une relation dialectique entre le pouvoir en place et le peuple, et dépend donc tant des volontés politiques publiques que des constructions intellectuelles et sexuelles personnelles. Cela se traduit concrètement, dans le domaine de la représentation, par la nécessité d'une double éducation publique et populaire. 


\section{Bibliographie}

Bazin, André. « Pour un cinéma impur. Défense de l'adaptation. »Qu'est-ce que le cinéma. Paris: Les Editions du cerf, 1952, 81-106.

Beaudry, Lucille. « L'art et le féminisme au Québec : aspects d'une contribution à l'interrogation politique. » Recherches féministes 272 (2014) : 7-19.

Bereni, Laure et Anne Revillard. « Des quotas à la parité : « féminisme d'État » et représentation politique (1974-2007). » Genèses 67 (2007) : 5-23.

Blottière, Mathilde. « Riad Sattouf : 'L'intrigue de 'Jacky' suit toutes les étapes de 'Cendrillon'. » Télérama. 28 janvier 2014. $<$ http://www.telerama.fr/cinema/riad-sattouf-1-intrigue-de-jacky-suittoutes-les-etapes-de-cendrillon-conte-de-la-domination-masculine-parexcellence, 108059.php>.

Bordwell David, Kristin Thompson et Jeremy Ashton. Film art: An introduction. New York: McGraw-Hill, 1997.

Braunstein, Jacques. « Riad Sattouf, sa vie est un roman (graphique). Interview de Riad Sattouf. GQ magazine. 2 février 2015. $<$ http://www.gqmagazine.fr/pop-culture/livres/articles/riad-sattouflarabe-du-futur-angouleme-fauve-d-or/23860>.

Brinaire, Jacques. " Regard dans le miroir inversé de Riad Sattouf. » Interview de Riad Sattouf. La Nouvelle République.fr. 26 janvier 2014. $<$ http://www.lanouvellerepublique.fr/FranceMonde/Loisirs/Cinema/n/ Contenus/Articles/2014/01/26/Regard-dans-le-miroir-inverse-deRiadSattouf-1772175>.

Butler, Judith. «Trouble dans le genre. Pour un féminisme de la subversion. » Population 61.3 (2006): 347-349.

Cavell, Stanley. The world Viewed: Reflections on the Ontology of Film. Cambridge: Harvard University Press, 1971.

Cojean, Annick. « Les noces barbares de Daech. » Le Monde, 13 déc. 2014.

Crenshaw, Kimberle. « Mapping the margins: Intersectionality, identity politics, and violence against women of color. » Stanford Law Review, 1991. 1241-1299.

Crenshaw, Kimberlé Williams. « Cartographies des marges : intersectionnalité, politique de l'identité et violences contre les femmes de couleur. " Trad. Oristelle Bonis. Cahiers du Genre 39 (1 février 2012) : 51-82.

Darré, Yann. « Esquisse d'une sociologie du cinéma. » Actes de la recherche en sciences sociales 161/162 (2006) : 122-136.

---. Histoire sociale du cinéma français. Paris : La Découverte, 2000.

Détrez, Christine. La Construction sociale du corps. Paris : Seuil, 2002.

Diken, Bülent et Carsten Bagge Lausten. Sociology Through the Projector. Abingdon-on-Thames: Routledge, 2008.

Esquenazi, Jean-Pierre. « Le film, un fait social. » Réseaux 18.99 (2000) :13-47.

Ferro, Marc. Analyse de film, analyse de sociétés. Paris: Hachette, 1975.

Firestone, Shulamith. The Dialectic of Sex: The Case for Feminist Revolution. New York : Morrow, 1970.

Fraser, Nancy et Estelle Ferrarese. Le féminisme en mouvements: des années 1960 à l'ère néolibérale. Paris : La Découverte, 2013.

Kaplan, Gisela. Contemporary Western European Feminism. S.1.. Routledge, 
2012.

Lever, Yves. L'analyse filmique. Montréal : Boréal, 1992.

Marrati, Paola. « Une image mouvante du scepticisme. » Rue Descartes 3 (2006): 62-70.

Maugue, Annelise. «L'Ève nouvelle et le vieil Adam. Identités sexuelles en crise. " Histoire des femmes en Occident 4 (2002) : 527-543.

Michard, Claire. Sexe en linguistique: Sémantique ou zoologie? Paris: L'Harmattan, 2002.

Morgan, Robin. Sisterhood is Powerful. New York: Random House, 1970.

Neveu, Érik et Armand Mattelart. Introduction aux cultural studies. Paris : La Découverte, 2010.

Panofsky, Erwin. La perspective comme forme symbolique et autres essais. Paris: Éditions de Minuit, 1975.

Riot-Sarcey, Michèle. Histoire du féminisme. Paris : La Découverte, 2010.

Sattouf, Riad. Interview. "L'arabe du futur. » Clique. 18 juin 2015.

Sorlin, Pierre. «Clio à l'écran, ou l'historien dans le noir. » Revue d'histoire contemporaine. juin 1974. 253-278.

Stetson, Dorothy McBride et Amy Mazur. Comparative State Feminism. S.1. Sage Publications, 1995.

Thébaud, Françoise. Écrire l'histoire des femmes et du genre. Lyon : ENS Éditions, 2007.

Zabunyan, Elvan. « Histoire de l'art contemporain et théories féministes : le tournant de 1970. » Cahiers du Genre 43 (2011) : 171-186. 